\title{
Neural mechanisms of atrial arrhythmias
}

Mark J. Shen, Eue-Keun Choi, Alex Y. Tan, Shien-Fong Lin, Michael C. Fishbein, Lan S. Chen, Peng-Sheng Chen

\begin{abstract}
The past 5 years have seen great advances in the knowledge of neural mechanisms of atrial arrhythmogenesis. Direct autonomic nerve recordings demonstrate that simultaneous sympathovagal discharges and intrinsic cardiac nerve activities are common triggers of paroxysmal atrial tachycardia and atrial fibrillation. While activity of the autonomous nervous system (ANS) is crucial in triggering paroxysmal atrial fibrillation, a high incidence of sympathovagal coactivation at baseline is associated with a high vulnerability to pacing-induced sustained atrial fibrillation, suggesting that ANS has a role in the development of persistent atrial fibrillation. Modulation of ANS activity may constitute an important therapeutic strategy for the management of atrial tachyarrhythmias. Specifically, continuous, low-level stimulation of the left cervical vagus nerve effectively suppresses atrial tachyarrhythmias by reducing the nerve activity of the stellate ganglion. Clinically, compared with pulmonary vein isolation alone, the addition of ablation of intrinsic cardiac ganglia may confer better outcomes for patients with paroxysmal atrial fibrillation. These findings suggest that further investigations in the neural mechanisms of atrial arrhythmias might lead to better management of patients with atrial arrhythmias. In this article, we review the role of the ANS in the induction and maintenance of atrial arrhythmias and the role of neural modulation as a treatment strategy for atrial arrhythmias.
\end{abstract}

Shen, M. J. et al. Nat. Rev. Cardiol. advance online publication XX Month 2011;

doi:10.1038/XXX 
Krannert Institute of Cardiology, Department of Medicine, Indiana University School of Medicine, 1801 North Capitol Avenue, E475, Indianapolis, IN 46202, USA (M. J. Shen, S.-F. Lin, P.-S. Chen). Department of Internal Medicine, University of Illinois at Chicago / Advocate Christ Medical Center, 4440 West 95th Street, 131 NO, Oak Lawn, Illinois 60453 (M. J. Shen). Department of Neurology, Indiana University School of Medicine, 545 Barnhill Drive, EH 125, Indianapolis, IN 46202, USA (L. S. Chen). Department of Internal Medicine, Seoul National University Hospital, 28 Yongon-dong, Chongno-gu Seoul 110-744, Republic of Korea (E.-K. Choi). Beth Israel Deaconess Medical Center, Harvard Medical School, 330 Brookline Avenue, Boston, MA 02215, USA (A. Y. Tan). Department of Pathology and Laboratory Medicine at David Geffen School of Medicine, University of California Los Angeles, 10833 Le Conte Avenue, Los Angeles, CA 90095, USA (M. C. Fishbein.).

\section{Correspondence to:}

\section{P.-S. Chen}

chenpp@iupui.edu

\section{Competing interests}

P.-S. Chen declares associations with the following companies: Medtronic and St Jude Medical. See the article online for full details of the relationships. The other authors declare no competing interests.

\section{Key points}

- Simultaneous sympathovagal discharges contribute to development and maintenance of atrial arrhythmias, as they increase calcium transient, trigger spontaneous calcium release from the sarcoplasmic reticulum, and shorten atrial action potentials 
- Direct nerve recordings in ambulatory animals have shown that paroxysmal atrial tachyarrhythmias are usually preceded by simultaneous sympathetic and vagal discharges and are invariably triggered by intrinsic cardiac nerve activities

- Studies in ambulatory dogs have shown that cardiac nerve activities are also important in sustained atrial fibrillation

- Novel strategies, including neural ablation and neural stimulation, can reduce arrhythmogenic nerve activities but warrant further studies before they can be widely applied in clinical settings

\section{Introduction}

Atrial fibrillation (AF) is the most common arrhythmia in developed countries and affects approximately 2.3 million people in the USA alone. ${ }^{1}$ AF is a complex disease that is initiated by a specific trigger and requires a vulnerable substrate for the maintenance of the disease. The pulmonary veins (PVs) are critically important in initiating $A F .^{2,3}$ Once initiated, $A F$ leads to atrial remodeling, through alteration of electrical and structural properties of the atria, and thus facilitates maintenance and recurrence of AF. However, the precise mechanism by which ectopic foci in PVs are initiated remains unclear. A possible mechanism involves paroxysmal discharges of the autonomous nervous system (ANS). Histological studies show that the PVs and the PV-left-atrial (LA) junction are richly innervated by both sympathetic and parasympathetic nerves. ${ }^{4,5}$ Enhanced activity of these intrinsic cardiac nerves has been implicated in triggering focal AF that arises from PVs. ${ }^{6,7}$ Ablation of these nerves suppresses or eliminates focal AF from PVs, ${ }^{8}$ supporting a causal relationship between the activity of intrinsic cardiac nerves and atrial arrhythmias. In this article, we review the role of the ANS in the induction and maintenance of atrial arrhythmias, and discuss neural modulation as a treatment strategy for patients with atrial arrhythmias. 


\section{Neuroanatomy of the heart}

Autonomic innervation of the heart

The cardiac ANS (Figure 1a) consists of extrinsic and intrinsic components. ${ }^{9,10}$ The sympathetic innervation comes from the superior cervical ganglia and the cervicothoracic (stellate) ganglia

(Figure $1 \mathrm{~b}$, left image), which, respectively, communicate with the cervical nerves $\mathrm{C} 1-\mathrm{C} 3$, and with the cervical nerves $\mathrm{C} 7-\mathrm{C} 8$ to the thoracic nerves T1-T2. In addition, the thoracic ganglia (as low as at least the $4^{\text {th }}$ thoracic ganglion) also contribute to the sympathetic innervation of the heart ${ }^{11}$. The superior, middle, and inferior cardiac nerves from these ganglia innervate the heart by following the brachiocephalic trunk, common carotid arteries, and subclavian arteries. The thoracic cardiac nerves in the posterior mediastinum follow a more complex course to reach the heart in the middle mediastinum. Parasympathetic innervation is mediated by the vagus nerves and divided into superior, middle, and inferior branches (Figure 1b, middle image). Most of the vagal nerve fibers converge at a distinct fat pad between the superior vena cava and the aorta (known as the 'third fat pad') en route to the sinus and atrioventricular nodes.

Autonomic innervation of the atria

In addition to the extrinsic nervous system, the heart is also innervated by an extensive intrinsic cardiac nervous system. Many investigators have studied the macroscopic and microscopic anatomy of cardiac autonomic nerves within the atria. Armour et al. provided a detailed map of the distribution of autonomic nerves in human hearts. ${ }^{13}$ They found that the intrinsic cardiac ANS forms a complex neural network composed of ganglionated plexi, which are concentrated within epicardial fat pads and the interconnecting ganglia and axons. ${ }^{13-15}$ The sinus node is primarily innervated by the right-atrial ganglionated plexi, whereas the atrioventricular node is innervated by the inferior vena cava-inferior atrial ganglionated plexi (at the junction of inferior 
vena cava and the left atrium). ${ }^{9,16-18}$ Another region that is richly innervated by the ANS and has a high density of ganglionated plexi is the PV-LA junction. The PV-LA junction contains closely located adrenergic and cholinergic nerves (Figure 1c,d). ${ }^{4}$ Stimulation of the ganglionated plexi at the PV-LA junction can convert the firing from PVs into AF in dogs ${ }^{19}$ and in humans. ${ }^{20}$ Increasing levels of stimulation of these plexi progressively reduce the atrial effective refractory period and widen the window of vulnerability to $A F .{ }^{21}$ On the basis of results from experiments involving acute ablation and neural stimulation in anesthetized dogs, Hou et al. postulated that these ganglionated plexi serve as 'integration centers' for the right and left vagosympathetic trunks, modulating cardiac electrophysiology and the inducibility of AF. ${ }^{18}$ Radiofrequency ablation at these sites can potentially result in successful denervation and prevent the inducibility of $A F .^{22,23}$ An experimental study in dogs indicates that ablation of the autonomic ganglia at the base of the PVs suppresses vagally induced $A F .{ }^{24}$ These observations suggest that damage to the atrial ganglionated plexi might contribute to the effectiveness of ablation procedures that are directed at PVs. Aside from the ganglionated plexi around the base of the PVs, several ganglionated plexi with only 2-10 neurons are located on the periphery of the atria. Similarly to the major ganglionated plexi, these peripheral plexi can become independently hyperactive, particularly when they are separated from the major ganglionated plexi as a result of acute injury or chronic scarring. ${ }^{13}$

\section{ANS and atrial tachyarrhythmias}

\section{Potential role of specialized conducting cells}

Sympathetic activation of the heart facilitates arrhythmogenesis by increasing calcium entry and the spontaneous release of calcium from the sarcoplasmic reticulum. ${ }^{25,26}$ Vagal activation of the heart can reduce the atrial effective refractory period, ${ }^{27}$ augment spatial electrophysiological heterogeneity of the atria ${ }^{28}$ and promote early afterdepolarizations towards the end of phase 3 in 
the cardiac action potential. ${ }^{29}$ Abnormal intracellular calcium handling and short action potential durations are key features of electrophysiological remodeling in AF. ${ }^{30}$ Sharifov and colleagues showed that acetylcholine infusion in the sinus-node artery invariably induced AF in dogs; acetylcholine-mediated AF was facilitated by the beta-adrenoceptor agonist isoproterenol, which decreased the threshold concentration of acetylcholine required for the induction of AF and prolonged episodes of AF. ${ }^{31}$ These results indicate that combined sympathovagal discharge has a strong profibrillatory effect in the atria. In addition, periodic-acid-Schiff (PAS)-positive cells are found along the intima of PV muscle sleeves. ${ }^{32}$ Since PAS staining is commonly used to identify cells with high glycogen content in the ventricles, such as Purkinje cells, ${ }^{33}$ the presence of PASpositive cells is consistent with the hypothesis that specialized conducting cells exist in the PVs. These PAS-positive cells are abundantly innervated by sympathetic nerves (identified by positive tyrosine-hydroxylase-staining) and located in high density at ectopic foci where focal atrial tachyarrhythmias arise. ${ }^{34}$ Sympathetic activation might trigger focal discharges from these cells. In addition to PAS-positive cells, other specialized cells exist in the PVs, such as melanocyte-like cells ${ }^{35}$ and CD117/c-kit-positive interstitial Cajal-like cells (ICLC). ${ }^{36,37}$ Cajal cells are pacemakers of the gastrointestinal muscle along the alimentary $\operatorname{tract}^{38}$ and initiate rhythmicity in the gastrointestinal tract through the spontaneous activation of inward calcium currents. Morel et al. found that two out of three patients with ICLCs in their PVs had a history of AF, but the investigators did not provide a detailed mechanism by which these cells contribute to atrial arrhythmias. ${ }^{37}$ The melanin-synthesis enzyme D-dopachrome tautomerase (DCT, also known as D-dopachrome decarboxylase) is involved in the regulation of intracellular calcium (melanin binds calcium with an affinity similar to calmodulin) and prevents the build-up of reactive melanin-synthesis intermediates in melanocytes. Levin et al. identified a unique DCTexpressing cell population in murine and human hearts that populated the PVs, atria, and atrioventricular canal. ${ }^{35}$ These cells express adrenergic and muscarinic receptors, and display 
transcriptional profiles that are distinct from dermal melanocytes. Mice lacking DCT demonstrate increased susceptibility to atrial arrhythmias. The dysfunction of melanocyte-like cells in the atrium and in the PVs might contribute to atrial arrhythmias when the ANS is activated. However, because none of these studies demonstrates a clear electrical connection between these specialized cells and cardiomyocytes, the extent to which melanocyte-like cells contribute to the automaticity and triggered activity in human PVs remains incompletely understood.

\section{Data from analyses of heart-rate variability}

As early as 1978, Coumel et al. reported that the activity of cardiac ANS might predispose patients to develop paroxysmal atrial arrhythmias. ${ }^{39}$ These researchers observed that vagal activity was a trigger for AF in young patients with normal hearts, whereas sympathetic activity was a trigger for AF in patients with organic heart disease. ${ }^{40}$ This finding is consistent with another study using analyses of heart-rate variability. ${ }^{41}$ Data from subsequent studies ${ }^{42-49}$ indicate that, rather than being triggered by either vagal or sympathetic activity, the onset of AF is associated with an imbalance between these two arms of the ANS. The same phenomenon might also trigger atrial flutter. ${ }^{50}$ In an analysis of over 1,500 participants of the Euro Heart Survey with paroxysmal $A F,{ }^{51}$ the onset of atrial arrhythmias was frequently preceded by autonomic triggers, including adrenergic triggers (associated with exercise or emotion), vagal triggers (during the night without any adrenergic trigger), and mixed adrenergic and vagal activations. However, in all these studies, ${ }^{39-51}$ ANS activity was evaluated indirectly, mostly by analyses of heart-rate-variability parameters on short-term electrocardiogram (ECG) recordings. Although analysis of heart-rate variability has been a popular, noninvasive way of assessing cardiac ANS activity, this technique has substantial limitations in that it measures only relative changes in ANS activity instead of the absolute intensity of sympathetic or parasympathetic discharges. Furthermore, analysis of heart-rate variability also requires an intact sinus node that 
mediates adequate cardiac responses to ANS. Experimentally, pacing-induced chronic AF disrupts sinus-node function and impairs the response of the sinus node to autonomic drives. ${ }^{52}$ Similar sinus-node dysfunction is also found in heart failure, which is commonly associated with atrial tachyarrhythmias. In the presence of heart failure, the power spectrum of heart-ratevariability analysis is heavily influenced by the diminished response of the sinus node to ANS activation and cannot be used to accurately gauge the underlying sympathetic tone. ${ }^{53}$ The same limitation of heart-rate-variability analysis might also apply to patients with AF, as the disease is often associated with sinus-node dysfunction. ${ }^{52,54}$ Therefore, analysis of heart-rate variability in patients with AF might not reliably reflect the underlying activity of the ANS. Direct recordings of ANS activity would be highly desirable to determine if ANS activity directly triggers atrial arrhythmias.

\section{Findings from nerve-activity recordings}

Paroxysmal atrial tachyarrhythmias

The first successful long-term, in vivo nerve recording was done by Barrett and colleagues, who recorded renal sympathetic nerve activity in conscious rabbits for more than 7 days and published their results in $2003 .{ }^{55}$ However, renal sympathetic nerve activity might not be representative of cardiac sympathetic nerve activity. In a pioneering study, Jung and colleagues continuously recorded the activity of stellate ganglia in healthy dogs using implanted radiotransmitters for an average of 41.5 days ( \pm 16.6 days).$^{56}$ The results showed the feasibility of chronic cardiac nerve recordings and demonstrated a circadian variation of sympathetic outflow to the heart. ${ }^{56}$ To further determine whether spontaneous ANS discharge can serve as a trigger of AF, subsequent studies have used intermittent, rapid atrial pacing to facilitate the development of paroxysmal atrial tachyarrhythmias. ${ }^{27}$ Rapid atrial pacing at either left or right atrial appendage causes not only electrical remodeling of the atria, ${ }^{27}$ but also significant neural 
remodeling that is characterized by a heterogeneous increase of sympathetic innervations ${ }^{57}$ and extensive nerve sprouting. ${ }^{58}$ Such neural remodeling may, in turn, promote the electrical remodeling caused by rapid atrial pacing. ${ }^{59}$ Tan et al. implanted a pacemaker and a radiotransmitter in dogs to simultaneously recorded nerve activities of the left stellate ganglion and left vagal nerve as well as a surface ECG over a period of several weeks. ${ }^{60}$ Intermittent rapid atrial pacing was performed and ANS activity was monitored when the pacemaker was turned off. Simultaneous sympathovagal discharge (Figure 2a) was found to be the mostfrequent trigger of paroxysmal atrial tachycardias and AF, and a common trigger for premature atrial contractions. ${ }^{60}$ Cryoablation of bilateral stellate ganglia and of the superior cardiac branches of the left vagus nerve eliminated all episodes of paroxysmal AF and atrial tachycardias, indicating a causal relationship between ANS activity and the generation of paroxysmal atrial tachyarrhythmias. Similarly, in a canine model of pacing-induced heart failure, simultaneous sympathovagal discharge was the most frequent trigger of atrial tachyarrhythmias, ${ }^{61}$ which could be prevented by cryoablation of the stellate ganglion and the T2-4 thoracic sympathetic ganglia. ${ }^{62}$ These findings are consistent with reports by Sharifov et al. $^{31}$ and Patterson et al., ${ }^{7}$ and further support the hypothesis that ANS activity is critically important in the generation of paroxysmal AF. More recently, Choi and colleagues took this technique a step further by recording ANS activity of intrinsic cardiac ganglia. ${ }^{63}$ In addition to the left stellate ganglion and the left thoracic vagal-nerve, nerve activities from the superior, left ganglionated plexi and the ligament of Marshall (Figure 1b, right panel) were monitored in dogs subjected to intermittent, rapid atrial pacing. The team found that discharge of intrinsic cardiac nerves from the superior, left ganglionated plexi and the ligament of Marshall was an invariable trigger of paroxysmal AF and atrial tachycardias (Figure $2 b$ ). This finding is supported by results of a study demonstrating the importance of ganglionated plexi around the PVs for AF as a result of atrial tachycardia remodeling. ${ }^{64}$ Another interesting observation of this study ${ }^{63}$ is that the 
activities of intrinsic cardiac nerves might 'contaminate' local atrial electrograms, resulting in recordings similar to that of complex fractionated atrial electrograms. This result echoes early experimental findings ${ }^{65,66}$ and a more-recent observation in patients with $A F{ }^{67}$ These findings may explain the clinical efficacy of ablative therapy that targets those intrinsic cardiac ganglia ${ }^{22,23,68}$ or sites with complex fractionated atrial electrograms. ${ }^{69}$

Sustained atrial fibrillation

Some, but not all, patients with paroxysmal AF progress to sustained forms of the disorder (known as 'persistent' and 'permanent' AF); in one study, roughly half of the patients with lone paroxysmal AF progressed to sustained AF after 25 years of follow-up. ${ }^{70}$ The time-toprogression varies considerably between individuals who do progress from paroxysmal to sustained AF. ${ }^{70}$ Reasons for such interindividual disparities in the susceptibility to progression of AF remain poorly understood. Intermittent rapid atrial pacing in large animals can initially induce paroxysmal $A F ;{ }^{60,63}$ if the rapid pacing continues, sustained AF will develop. ${ }^{27}$ Wijffels et al. proposed that AF begets AF through progressive, pacing-induced electrical remodeling, such as shortening of the effective refractory periods. ${ }^{27}$ However, the researchers also noted that the time course of changes in atrial refractoriness did not exactly parallel the development of sustained AF, indicating that other factors might have a role in the progression to sustained AF. To determine whether autonomic nerve activity influences the development of sustained AF, we analyzed long-term recordings of nerve activities in ambulatory dogs and determined the duration of intermittent rapid atrial pacing needed to induce sustained ( $>48 \mathrm{~h}$ ) AF. ${ }^{71}$ We found that differential patterns of interactions among cardiac autonomic structures naturally exist before pacing (Figure 3), and that distinct patterns of autonomic interactions between sympathetic and vagal nerves are associated with a faster development of sustained AF. Specifically, compared with the majority (75\%) of studied dogs, who had nonlinear or L-shaped 
sympathovagal correlation (Figure 3a), ambulatory dogs with good linear sympathovagal correlation and higher vagal tone at baseline (Figures 3b) had more paroxysmal atrial tachycardias at baseline and faster induction of sustained AF by rapid atrial pacing. Figure 3c shows that, in a dog with an L-shaped sympathovagal correlation, sympathetic and vagal discharges activated alternatively. During phases of lone sympathetic nerve activation the heart rate increased. Vagal nerve activation alone decreased the heart rate. By contrast, Figure 3d illustrates an example of concomitant sympathovagal firings that led to heart-rate acceleration in a dog that displayed a linear sympathovagal correlation. These findings indicate that differential patterns of autonomic discharges that are naturally present in dogs before atrial pacing have significant implications on cardiac arrhythmogenesis. Simultaneous sympathovagal discharges are not only important in triggering paroxysmal atrial tachyarrhythmias (including AF), ${ }^{60,61}$ but might also promote the development of sustained AF. Different forms of sympathovagal discharge patterns might also be present in human patients under normal physiological circumstances, and differential ANS discharge patterns may predetermine which patients will be at greater risks of progression from paroxysmal to sustained AF.

\section{Controlling atrial fibrillation}

\section{Ablation of intrinsic cardiac nerves}

Radiofrequency catheter-ablation

In their seminal paper published in 1998 , Haissaguerre et al. showed that AF can emerge as a result of depolarizations in the PV and established the fundamental role of ablation procedures directed to these veins in the treatment of patients with AF. ${ }^{2}$ Elvan and colleagues had demonstrated that radiofrequency catheter ablation of the atria can eliminate pacing-induced, sustained AF by denervation of efferent vagal nerves of the atria. ${ }^{72,73}$ Because linear lesions from standard PV-directed ablation run through areas with high concentrations of ganglionated 
plexi, ${ }^{74}$ autonomic modification or denervation might be a potential mechanism that contributes to the effectiveness of such ablation procedures. ${ }^{23,24}$ Autonomic denervation targeting the ganglionated plexi around the base of the PVs has been proposed as an adjunctive procedure ${ }^{22,75-78}$ or even as a stand-alone treatment for patients with AF. ${ }^{68,79,80}$ However, the effectiveness of autonomic modulation as an adjunctive therapeutic strategy to catheter ablation in this setting has been inconsistent. ${ }^{22,23,78,81,82}$ Pappone et al. achieved vagal denervation by abolishing all evoked vagal reflexes around PVs in 34.3\% of patients with paroxysmal AF who also underwent circumferential PV ablation. ${ }^{23}$ Vagal withdrawal was evident for up to 3 months after the procedure and these patients were significantly less likely to have recurrent AF than those whose vagal reflexes could not be evoked during the procedure. Results of a study by Scherlag and colleagues showed that the ablation of ganglionated plexi in addition to PV antrum isolation increased ablation success (meaning AF did not recur) from $70 \%$ to $91 \%$ among patients with paroxysmal or persistent AF after 12 months of follow-up. ${ }^{78}$ In a small number of patients who had vagal-induced paroxysmal AF, attempted denervation alone without substrate modification in the atria effectively prevented the recurrence of AF in two out of 11 patients. ${ }^{79}$ These two patients also had the most pronounced and persistent changes in heart-rate variability. By contrast, Lemery et al. found that freedom of recurrent AF during 8 months of follow-up was observed in only $50 \%$ of patients with paroxysmal and persistent AF who underwent combined ablation of PVs and ganglionated plexi guided by endocardial highfrequency stimulation. ${ }^{81}$ These conflicting findings suggest that the interactions between the ANS and AF are complex and currently poorly understood. Individual variability in the autonomic triggers might account for these discrepancies, with some patients having more pronounced autonomic triggers than others. ${ }^{51}$ Alternatively, catheter-ablation procedures might have failed to effectively eliminate all intrinsic cardiac nerves in the atria. The ideal sites to ablate continue to be debated. Pokushalov and colleagues demonstrated that an anatomic, regionally extensive 
approach for the ablation of ganglionated plexi confers better clinical outcomes than selective ablation of ganglionated plexi directed by high-frequency stimulation. ${ }^{68}$ In animal studies, partial vagal denervation of the high right atrium increased the inducibility of AF. ${ }^{83}$ Together, these studies suggest that thorough denervation is important in achieving best clinical outcomes. However, radiofrequency ablation in patients is followed by an elevation in the concentration of nerve growth factor in peripheral veins; ${ }^{84}$ this increase might cause nerve sprouting and sympathetic hyperinnervation. ${ }^{85}$ More-thorough ablation may thus lead to more nerve sprouting. Furthermore, vagal effects can be restored within 4 weeks of ablation. ${ }^{86}$ Despite these potential limitations, data from one study demonstrate that the beneficial effects of an ablation of ganglionated plexi continue to increase for 12 months after ablation. ${ }^{87}$ Investigators of a morerecent, randomized, multicenter trial (albeit of small scale) showed that patients who underwent ablation of ganglionated plexi and isolation of PVs had less recurrences than patients who underwent PV isolation alone within 12 months after ablation. ${ }^{75}$ These preliminary studies suggest that, despite neural remodeling, the destruction of neurons within the ganglionated plexi by radiofrequency catheter-ablation techniques might have positive long-term effects.

Novel denervation methods

Over the past 2 years, other methods of atrial autonomic denervation have been developed. Oh et al. showed that local injection of botulinum toxin into epicardial fat pads can accomplish shortterm autonomic denervation (for about $5 \mathrm{~h}$ ) and suppress AF induced by bilateral vagus-nerve stimulation. ${ }^{88}$ The investigators hypothesized that this short-term block of ganglionated plexi might be applicable in certain clinical occasions, such as the prevention of AF after cardiac surgery. Similarly, Stavrakis et al. ${ }^{89}$ showed that a local injection of vasostatin-1 (a cardioregulatory peptide with strong antiadrenergic effects that are mediated via the release of nitric oxide) into the epicardial fat pads can suppress AF induced by a single extra electrical 
stimulus. ${ }^{89}$ Yu et al. demonstrated that magnetically guided particles that contain a neurotoxin can be used to selectively inhibit ganglionic tissue, without affecting atrial tissue. ${ }^{90}$ These techniques might provide alternative, less-invasive strategies for radiofrequency ablation, especially for patients with prominent autonomic triggers in whom modulation of autonomic nerves alone may be sufficient to control their atrial fibrillation.

\section{Ablation of extrinsic cardiac nerves}

Ablation of the left stellate ganglion is known to reduce the incidence of ventricular arrhythmias in patients with long-QT syndrome, ${ }^{91,92}$ but the technique has been less-vigorously investigated in the clinic for the treatment of atrial tachyarrhythmias. In a canine model of intermittent rapid atrial pacing, ablation of the stellate ganglion and cardiac branches of the left vagal nerve eliminated all episodes of paroxysmal atrial tachyarrhythmias. ${ }^{60}$ The same procedure also prevented atrial tachyarrhythmias in ambulatory dogs with pacing-induced heart failure. ${ }^{62}$ These findings support the argument that ablation of extrinsic cardiac nerves might be an effective alternative therapy to the ablation of intrinsic cardiac ganglia for the treatment of AF.

\section{Neural stimulation}

Acupuncture

Acupuncture has been used for thousands of years to treat humans with various diseases, including hypertension, coronary artery disease, arrhythmias, and myocardial infarction. One effect of acupuncture is the modulation of autonomic nerves. ${ }^{93}$ For example, stimulating an acupuncture point in the forearm (such as the Neiguan or Nei-Kuan acupoint) might lead to vagomimetic and sympatholytic actions. ${ }^{94,95}$ Alternatively, through its antisympathetic effect, stimulation of the median afferent nerve (corresponding to the Neiguan acupoint) improves myocardial ischemia caused by reflexively induced sympathetic excitation in cats. ${ }^{96}$ 
Transcutaneous acupuncture of the forearm at the Ximen acupoint can also elicit vagal responses and reduce heart rate. ${ }^{97}$ These studies indicate that the inhibition of sympathetic activity and augmentation of vagal activity might in part underlie the beneficial effects of acupuncture. In a recent, small-scale clinical trial, acupuncture achieved an AF recurrence rate after cardioversion similar to amiodarone treatment, and lower than in acupuncture-sham and control groups. ${ }^{98}$ Further mechanistic and clinical studies are needed before acupuncture can be recommended for patients with AF.

\section{Electrical stimulation to peripheral nerves}

Abundant data exist to support the hypothesis that direct stimulation of a central or a peripheral nerve structure might reduce ventricular tachyarrhythmias. Chai et al. report that ventricular arrhythmias can occur after occluding the common carotid arteries in cats, most likely owing to the activation of the sympathetic nervous system. ${ }^{99}$ These arrhythmias can be augmented or inhibited by stimulating certain areas in the hypothalamus or mesencephalon. Peroneal nerve stimulation was also used to control ventricular arrhythmias associated with high sympathetic tone in experimental animals. ${ }^{100,101}$ Chronic vagus-nerve stimulation has been shown prevent ventricular fibrillation and sudden cardiac death in conscious dogs with a healed myocardial infarction. $^{102}$

Much more recently (in 2009 and 2010), neural stimulation was also shown to be antiarrhythmic at the atrial level. Although vagus-nerve stimulation is a reliable technique used for the experimental induction of AF, low-level cervical vagus-nerve stimulation (LL-VNS) at a stimulus strength $1 \mathrm{~V}$ below the threshold needed to reduce heart rate can lower intrinsic cardiac nerve activity and, paradoxically, suppress electrically induced AF in open-chest, anesthetized dogs. ${ }^{103,104}$ This suppression can be achieved even if the stimulus strength is $50 \%$ below the threshold, and LL-VNS reverses the electrical remodeling caused by rapid atrial 
pacing. ${ }^{104,105}$ Shen and colleagues used direct nerve recordings to demonstrate that continuous LL-VNS suppressed paroxysmal atrial tachyarrhythmias in ambulatory, conscious dogs through the reduction of stellate-ganglion nerve activity (Figure 4a). ${ }^{106}$ This reduction was most apparent (35\% lower than baseline activity) in the early morning (Figure 4b), when the incidence of both symptomatic, paroxysmal $\mathrm{AF}^{107}$ and sudden cardiac death is highest. ${ }^{108}$ After the cessation of LL-VNS, stellate-ganglion nerve activity gradually normalized to approximately its baseline level (Figure 4a). This 'washout effect' might validate the causal relationship between LL-VNS and the suppression of cardiac sympathetic outflow. Histologically, a significant reduction of tyrosine-hydroxylase-positive nerves and an increase of tyrosine-hydroxylase-negative ganglion cells in the left stellate ganglion were also observed (Figure 4c). In dogs subjected to rapid atrial pacing, incidences of both paroxysmal AF and atrial tachycardias were significantly lower during LL-VNS sessions than during sham-stimulation sessions. The functional and structural remodeling of the heart might explain the antiarrhythmic effects of LL-VNS at the atrial level. Transcutaneous stimulation at the tragus, the anterior protuberance of the outer ear, is shown to have effects similar to vagus-nerve stimulation and is less invasive than LL-VNS. ${ }^{109}$ Tragus stimulation has been applied in experimental animals to control atrial fibrillation. ${ }^{110}$

In summary, these data suggest that neural stimulation may be a promising treatment strategy for patients with AF. However, further mechanistic and clinical studies are warranted before a wider application of neural stimulation can be recommended.

Electrical stimulation of the spinal cord

A major limitation of the ablation or stimulation of peripheral nerves is that these nerves, including the extrinsic and intrinsic cardiac nervous system, have a diversity of functions and neurochemical activities. For example, adrenergic and cholinergic nerves are closely located in the ganglionated plexi at the PV-LA junction in humans. ${ }^{4}$ Many ganglion cells $(30 \%)$ have a dual 
adrenocholinergic phenotype. ${ }^{4}$ The thoracic vagal nerves also contain both adrenergic and cholinergic nerve fibers. ${ }^{61}$ In addition to the presence of both arms of the ANS in ganglionated plexi, these structures are also known to contain efferent, afferent, and local circuit neurons. ${ }^{10}$ Coordination of these nerve activities depends on the complex and interdependent feedback networks that make up the cardiac nervous system. ${ }^{111}$ This high functional and neurochemical diversity complicates the interpretation of the nerve activities recorded in ambulatory animals. In addition, because of the co-localization of both arms of ANS within the same nerve structure (the ganglionated plexi), selective stimulation or ablation of the efferent arms of either ANS component is technically difficult, if not impossible.

An alternative approach is to target the specific nexus points within the associated neural networks to achieve therapeutic effects..$^{24,112-115}$ Spinal-cord stimulation, for example, enhances parasympathetic activity ${ }^{116}$ and reduces the capacity of intrathoracic extracardiac neurons to transduce myocardial ischemia. ${ }^{112}$ This method also improves ventricular function and reduces ventricular arrhythmias in a canine model of heart failure after myocardial infarction. ${ }^{117}$ Spinalcord stimulation is also known to be effective in managing patients with drug-refractory angina pectoris. ${ }^{118,119}$ A multicenter, prospective clinical trial entitled Determining the Feasibility of Spinal Cord Neurmodulation for the Treatment of Chronic Heart Failure (DEFEAT-HF) has been started to evaluate the effects of spinal-cord stimulation in human patients with severe heart failure symptoms. ${ }^{120}$ Further studies are needed to determine if spinal-cord stimulation can reduce atrial tachyarrhythmias in humans.

\section{Conclusions}

Cardiac ANS activation can lead to significant changes in the atrial electrophysiology and facilitates both the initiation and maintenance of atrial arrhythmias. Although the mechanisms of arrhythmogenesis and the benefits of autonomic modulation remain to be fully clarified, direct 
measurement of cardiac, autonomic nerve activities has shown that the ANS is important, not only in triggering paroxysmal AF, but also in facilitating the development of persistent AF. Modulation of cardiac ANS outflow through either neural ablation or electrical neural stimulation might provide effective control for these atrial arrhythmias in the future.

\section{Review criteria}

The initial literature review was conducted using the PubMed database and the search terms "autonomic nervous system", "ganglionated plexi", "sympathetic", "vagal", "neural mechanisms", "atrial fibrillation", "arrhythmia", "ablation" and "acupuncture". Full-text articles published in English from 1970-2011 were included. Reference lists of comprehensive review articles were further examined for additional references. To bring the review up to date, we have included abstracts presented in the Heart Rhythm Society Annual Scientific Sessions in the first week of May 2011. Some of these abstracts provide important information on the feasibility of using neural modulation to prevent AF.

\section{Acknowledgements}

This study was supported in part by NIH Grants P01HL78931, R01HL78932, R01HL71140, R21HL106554, a Heart Rhythm Society Fellowship in Cardiac Pacing and Electrophysiology (M. J. Shen) and a Medtronic-Zipes Endowment (P. -S. Chen).

\section{References}

Figure 1 | Autonomic innervation of the heart. a | The cardiac sympathetic ganglia consist of cervical ganglia, stellate (cervicothoracic) ganglia, and thoracic ganglia. Parasympathetic innervation comes from the vagus nerves. $\mathrm{b}$ | Photographs of the left stellate ganglion (left image) and the superior cardiac branch of the left vagal nerve (middle image) in the dog heart. 
The LOM originates from the coronary sinus and connects to the LSPV; the SLGP is located between the LAA and the LSPV (right image). c | Co-localization of adrenergic and cholinergic nerves in the intrinsic cardiac ganglia. The same ganglion contained cholinergic ganglion cells (arrow, left image) and adrenergic nerve fibers (arrow, right image). d | Fluorescent dual-labeling of TH and ChAT, visualized by confocal microscopy, showed highly co-located ChAT-positive and TH-positive nerves within the intrinsic cardiac ganglia. Abbreviations: ChAT, choline acetyltransferase; LAA, left-atrial appendage; LIPV, left inferior pulmonary vein; LOM, ligament of Marshall; LSPV, left superior pulmonary vein; PA, pulmonary artery; SLGP, superior left ganglionated plexi; TH, tyrosine hydroxylase. Panel b reprinted with permission from American Heart Association (C Choi, E. K. et al. Circulation 121, 2615-2623 (2010). Panels C,d reprinted with permission from Elsevier Inc. (C) Tan, A. Y. et al. J. Am. Coll. Cardiol. 48, 132-143 (2006).

Figure 2 | The autonomic nervous system and paroxysmal atrial tachyarrhythmias. a | During an episode of atrial tachycardia, simultaneous sympathetic (blue arrowheads) and parasympathetic (green arrowheads) coactivation resulted in the conversion of atrial tachycardia to atrial fibrillation. b | In an episode of paroxysmal atrial tachycardia, the intrinsic nerve activities from the ligament of Marshall and superior left ganglionated plexi (red arrowheads) increased before a rise in extrinsic nerve activities from the stellate ganglion (blue arrowhead) and from the vagus nerve (green arrowhead). The magnified pseudo-ECG (right) shows the different P-wave morphologies during sinus rhythm (1) and during paroxysmal atrial tachycardia (2). Abbreviations: ECG, electrocardiogram, LOMNA, Ligament-of-Marshall nerve activity; SGNA, stellate ganglion nerve activity; SLGPNA, superior-left-ganglionated-plexi nerve activity; VNA, vagal nerve activity. Panel a reprinted with permission from American Heart Association ${ }^{\circ}$ Tan, A. Y. et al. Circulation, 118, 918-925 (2008) and panel b (c Choi, E. K. et al. Circulation 121, 2615-2623 (2010). 
Figure 3 | ANS and persistent atrial fibrillation. a | SGNA-VNA scatter plot from dog that was representative for $75 \%$ of dogs studied. The sympathovagal scatter plot shows a characteristic L-shaped pattern. b | Representative SGNA-VNA scatter plot of a dog with much faster induction of persistent atrial fibrillation after rapid atrial pacing. In this linear sympathovagal scatter plot, each dot represents an SGNA-VNA pair of nerve activity integrated over 1 min (total of 1440 data points over 24 h). c | An example of a recording from the same dog as in panel a, showing that simultaneous increases in VNA and SLGPNA (black arrows) resulted in heart-rate deceleration. $d$ | An example of simultaneous sympathovagal co-activation (black arrows) causing an acceleration of the heart rate in the same dog as in panel b. Abbreviations: ECG, electrocardiogram; LOMNA, Ligament-of-Marshall nerve activity; SGNA, stellate-ganglion nerve activity; VNA, vagal nerve activity; SLGPNA, superior-left-ganglionated-plexi nerve activity. Reprinted from Heart Rhythm 8(4), Shen et al. 583-589 (2011) with permission from Elsevier.

Figure 4 | LL-VNS and nerve activity of the left stellate ganglion. a | Continuous left-sided LL-VNS significantly reduced left stellate ganglion nerve activity over $24 \mathrm{~h}$. Nerve activity normalized to baseline level after cessation of LL-VNS. b | Hourly averages of stellate-ganglion nerve activity show that the reduction in integrated stellate-ganglion nerve activity was particularly striking in the early morning. c | TH immunostaining of left stellate ganglion of a healthy dog (upper image) and of a dog with LL-VNS (lower image). In dogs with LL-VNS, the density of TH-positive nerve structures in the left stellate ganglion is decreased, whereas that of ganglion cells lacking immunoreactivity to $\mathrm{TH}$ (arrows) is increased. ${ }^{*} P<0.05$. Abbreviations: LL-VNS, low-level vagus-nerve stimulation; SGNA, stellate-ganglion nerve activity $\mathrm{TH}$, tyrosine hydroxylase Reprinted with permission from American Heart Association @ Shen et al. Circulation 123, 2204-2212 (2011). 
1. Benjamin, E.J., et al. Prevention of atrial fibrillation: report from a national heart, lung, and blood institute workshop. Circulation 119, 606-618 (2009).

2. Haissaguerre, M., et al. Spontaneous initiation of atrial fibrillation by ectopic beats originating in the pulmonary veins. N.Engl.J Med. 339, 659-666 (1998).

3. Chen, S.A., et al. Initiation of atrial fibrillation by ectopic beats originating from the pulmonary veins: electrophysiological characteristics, pharmacological responses, and effects of radiofrequency ablation. Circulation 100, 1879-1886 (1999).

4. Tan, A.Y., et al. Autonomic innervation and segmental muscular disconnections at the human pulmonary vein-atrial junction: implications for catheter ablation of atrialpulmonary vein junction. Journal of the American College of Cardiology 48, 132-143 (2006).

5. Chou, C.C., et al. Intracellular calcium dynamics and anisotropic reentry in isolated canine pulmonary veins and left atrium. Circulation 111, 2889-2297 (2005).

6. Patterson, E., Po, S.S., Scherlag, B.J. \& Lazzara, R. Triggered firing in pulmonary veins initiated by in vitro autonomic nerve stimulation. Heart Rhythm 2, 624-631 (2005).

7. Patterson, E., et al. Sodium-calcium exchange initiated by the Ca2+ transient: an arrhythmia trigger within pulmonary veins. J Am Coll.Cardiol. 47, 1196-1206 (2006).

8. Lu, Z., et al. Autonomic Mechanism for Initiation of Rapid Firing from Atria and Pulmonary Veins: Evidence by Ablation of Ganglionated Plexi. Cardiovasc.Res. (2009).

9. Armour, J.A. Cardiac neuronal hierarchy in health and disease. Am.J.Physiol Regul.Integr.Comp Physiol 287, R262-R271 (2004).

10. Armour, J.A. Functional anatomy of intrathoracic neurons innervating the atria and ventricles. Heart Rhythm 7, 994-996 (2010). 
11. Kawashima, $\mathrm{T}$. The autonomic nervous system of the human heart with special reference to its origin, course, and peripheral distribution. Anat.Embryol.(Berl) 209, 425438 (2005).

12. Chiou, C.W., Eble, J.N. \& Zipes, D.P. Efferent vagal innervation of the canine atria and sinus and atrioventricular nodes--the third fat pad. Circulation 95, 2573-2584 (1997).

13. Armour, J.A., Murphy, D.A., Yuan, B.X., Macdonald, S. \& Hopkins, D.A. Gross and microscopic anatomy of the human intrinsic cardiac nervous system. Anat.Rec. 247, 289-298 (1997).

14. Yuan, B.X., Ardell, J.L., Hopkins, D.A., Losier, A.M. \& Armour, J.A. Gross and microscopic anatomy of the canine intrinsic cardiac nervous system. Anat.Rec. 239, 7587 (1994).

15. Pauza, D.H., Skripka, V. \& Pauziene, N. Morphology of the intrinsic cardiac nervous system in the dog: a whole-mount study employing histochemical staining with acetylcholinesterase. Cells Tissues.Organs 172, 297-320 (2002).

16. Randall, W.C., Milosavljevic, M., Wurster, R.D., Geis, G.S. \& Ardell, J.L. Selective vagal innervation of the heart. Ann Clin Lab Sci 16, 198-208 (1986).

17. Nakajima, K., Furukawa, Y., Kurogouchi, F., Tsuboi, M. \& Chiba, S. Autonomic control of the location and rate of the cardiac pacemaker in the sinoatrial fat pad of parasympathetically denervated dog hearts. J.Cardiovasc.Electrophysiol. 13, 896-901 (2002).

18. Hou, Y., et al. Ganglionated plexi modulate extrinsic cardiac autonomic nerve input: effects on sinus rate, atrioventricular conduction, refractoriness, and inducibility of atrial fibrillation. J.Am.Coll.Cardiol. 50, 61-68 (2007).

19. Scherlag, B.J., Yamanashi, W., Patel, U., Lazzara, R. \& Jackman, W.M. Autonomically induced conversion of pulmonary vein focal firing into atrial fibrillation. J.Am.Coll.Cardiol. 45, 1878-1886 (2005). 
20. Lim, P.B., et al. Intrinsic Cardiac Autonomic Stimulation Induces Pulmonary Vein Ectopy and Triggers Atrial Fibrillation in Humans. J Cardiovasc Electrophysiol (2011).

21. Zhou, J., et al. Gradients of atrial refractoriness and inducibility of atrial fibrillation due to stimulation of ganglionated plexi. J.Cardiovasc. Electrophysiol. 18, 83-90 (2007).

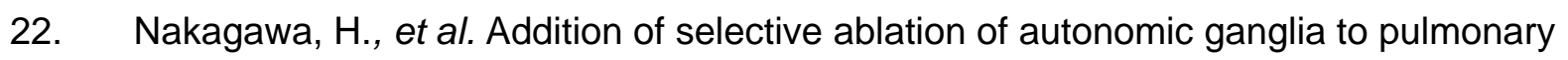
vein antrum isolation for treatment of paroxysmal and persistent atrial fibrillation. Circulation 110 III-459 (2006).

23. Pappone, C., et al. Pulmonary vein denervation enhances long-term benefit after circumferential ablation for paroxysmal atrial fibrillation. Circulation 109, 327-334 (2004).

24. Lemola, K., et al. Pulmonary vein region ablation in experimental vagal atrial fibrillation: role of pulmonary veins versus autonomic ganglia. Circulation 117, 470-477 (2008).

25. ter Keurs, H.E. \& Boyden, P.A. Calcium and arrhythmogenesis. Physiol Rev. 87, 457506 (2007).

26. Bers, D.M. Cardiac excitation-contraction coupling. Nature 415, 198-205 (2002).

27. Wijffels, M.C., Kirchhof, C.J., Dorland, R. \& Allessie, M.A. Atrial fibrillation begets atrial fibrillation. A study in awake chronically instrumented goats. Circulation 92, 1954-1968 (1995).

28. Fareh, S., Villemaire, C. \& Nattel, S. Importance of refractoriness heterogeneity in the enhanced vulnerability to atrial fibrillation induction caused by tachycardia-induced atrial electrical remodeling. Circulation 98, 2202-2209 (1998).

29. Burashnikov, A. \& Antzelevitch, C. Reinduction of atrial fibrillation immediately after termination of the arrhythmia is mediated by late phase 3 early afterdepolarizationinduced triggered activity. Circulation 107, 2355-2360 (2003).

30. Allessie, M.A., et al. Pathophysiology and prevention of atrial fibrillation. Circulation 103, 769-777 (2001). 
31. Sharifov, O.F., et al. Roles of adrenergic and cholinergic stimulation in spontaneous atrial fibrillation in dogs. J Am.Coll.Cardiol. 43, 483-490 (2004).

32. Perez-Lugones, A., et al. Evidence of specialized conduction cells in human pulmonary veins of patients with atrial fibrillation. J Cardiovasc. Electrophysiol. 14, 803-809 (2003).

33. Block, M.I., Said, J.W., Siegel, R.J. \& Fishbein, M.C. Myocardial myoglobin following coronary artery occlusion. An immunohistochemical study. Am.J Pathol. 111, 374-379 (1983).

34. Tan, A.Y., et al. Ectopic atrial arrhythmias arising from canine thoracic veins during in vivo stellate ganglia stimulation. Am J Physiol Heart Circ Physiol 295, H691-H698 (2008).

35. Levin, M.D., et al. Melanocyte-like cells in the heart and pulmonary veins contribute to atrial arrhythmia triggers. J.Clin.Invest (2009).

36. Gherghiceanu, M., et al. Interstitial Cajal-like cells (ICLC) in myocardial sleeves of human pulmonary veins. J.Cell Mol.Med. 12, 1777-1781 (2008).

37. Morel, E., Meyronet, D., Thivolet-Bejuy, F. \& Chevalier, P. Identification and distribution of interstitial Cajal cells in human pulmonary veins. Heart Rhythm. 5, 1063-1067 (2008).

38. Langton, P., Ward, S.M., Carl, A., Norell, M.A. \& Sanders, K.M. Spontaneous electrical activity of interstitial cells of Cajal isolated from canine proximal colon. Proc Natl Acad Sci U S A 86, 7280-7284 (1989).

39. Coumel, P., et al. The atrial arrhythmia syndrome of vagal origin. Arch Mal Coeur Vaiss 71, 645-656 (1978).

40. Coumel, P. Autonomic influences in atrial tachyarrhythmias. Journal of Cardiovascular Electrophysiology 7, 999-1007 (1996).

41. Huang, J.L., Wen, Z.C., Lee, W.L., Chang, M.S. \& Chen, S.A. Changes of autonomic tone before the onset of paroxysmal atrial fibrillation. International Journal of Cardiology 66, 275-283 (1998). 
42. Fioranelli, M., et al. Analysis of heart rate variability five minutes before the onset of paroxysmal atrial fibrillation. Pacing. Clin.Electrophysiol 22, 743-749 (1999).

43. Tomita, T., et al. Role of autonomic tone in the initiation and termination of paroxysmal atrial fibrillation in patients without structural heart disease. J Cardiovasc Electrophysiol 14, 559-564 (2003).

44. Dimmer, C., et al. Variations of autonomic tone preceding onset of atrial fibrillation after coronary artery bypass grafting. American Journal of Cardiology 82, 22-25 (1998).

45. Coccagna, G., Capucci, A., Bauleo, S., Boriani, G. \& Santarelli, A. Paroxysmal atrial fibrillation in sleep. Sleep 20, 396-398 (1997).

46. Herweg, B., Dalal, P., Nagy, B. \& Schweitzer, P. Power spectral analysis of heart period variability of preceding sinus rhythm before initiation of paroxysmal atrial fibrillation. Am.J.Cardiol. 82, 869-874 (1998).

47. Zimmermann, M. \& Kalusche, D. Fluctuation in autonomic tone is a major determinant of sustained atrial arrhythmias in patients with focal ectopy originating from the pulmonary veins. J.Cardiovasc. Electrophysiol. 12, 285-291 (2001).

48. Bettoni, M. \& Zimmermann, M. Autonomic tone variations before the onset of paroxysmal atrial fibrillation. Circulation 105, 2753-2759 (2002).

49. Amar, D., Zhang, H., Miodownik, S. \& Kadish, A.H. Competing autonomic mechanisms precede the onset of postoperative atrial fibrillation. J.Am.Coll.Cardiol. 42, 1262-1268 (2003).

50. Wen, Z.C., Chen, S.A., Tai, C.T., Huang, J.L. \& Chang, M.S. Role of autonomic tone in facilitating spontaneous onset of typical atrial flutter. J.Am.Coll.Cardiol. 31, 602-607 (1998).

51. de Vos, C.B., et al. Autonomic trigger patterns and anti-arrhythmic treatment of paroxysmal atrial fibrillation: data from the Euro Heart Survey. Eur Heart J 29, 632-639 (2008). 
52. Elvan, A., Wylie, K. \& Zipes, D.P. Pacing-induced chronic atrial fibrillation impairs sinus node function in dogs--electrophysiological remodeling. Circulation 94, 2953-2960 (1996).

53. Piccirillo, G., et al. Power spectral analysis of heart rate variability and autonomic nervous system activity measured directly in healthy dogs and dogs with tachycardiainduced heart failure. Heart Rhythm. 6, 546-552 (2009).

54. Gomes, J.A., Kang, P.S., Matheson, M., Gough, W.B., Jr. \& El Sherif, N. Coexistence of sick sinus rhythm and atrial flutter-fibrillation. Circulation 63, 80-86 (1981).

55. Barrett, C.J., et al. What sets the long-term level of renal sympathetic nerve activity: a role for angiotensin II and baroreflexes? Circ.Res. 92, 1330-1336 (2003).

56. Jung, B.C., et al. Circadian variations of stellate ganglion nerve activity in ambulatory dogs. Heart Rhythm 3, 78-85 (2006).

57. Jayachandran, J.V., et al. Atrial fibrillation produced by prolonged rapid atrial pacing is associated with heterogeneous changes in atrial sympathetic innervation. Circulation 101, 1185-1191 (2000).

58. Chang, C.M., et al. Nerve sprouting and sympathetic hyperinnervation in a canine model of atrial fibrillation produced by prolonged right atrial pacing. Circulation 103, 22-25 (2001).

59. Lu, Z., et al. Atrial fibrillation begets atrial fibrillation: autonomic mechanism for atrial electrical remodeling induced by short-term rapid atrial pacing. Circ Arrhythm Electrophysiol 1, 184-192 (2008).

60. Tan, A.Y., et al. Neural mechanisms of paroxysmal atrial fibrillation and paroxysmal atrial tachycardia in ambulatory canines. Circulation 118, 916-925 (2008).

61. Ogawa, M., et al. Left stellate ganglion and vagal nerve activity and cardiac arrhythmias in ambulatory dogs with pacing-induced congestive heart failure. Journal of the American College of Cardiology 50, 335-343 (2007). 
62. Ogawa, M., et al. Cryoablation of stellate ganglia and atrial arrhythmia in ambulatory dogs with pacing-induced heart failure. Heart Rhythm 6, 1772-1779 (2009).

63. Choi, E.-K., et al. Intrinsic cardiac nerve activity and paroxysmal atrial tachyarrhythmia in ambulatory dogs. Circulation 121, 2615-2623 (2010).

64. Nishida, K., et al. The role of pulmonary veins vs. autonomic ganglia in different experimental substrates of canine atrial fibrillation. Cardiovasc Res 89, 825-833 (2011).

65. Lin, J., et al. Autonomic mechanism to explain complex fractionated atrial electrograms (CFAE). J.Cardiovasc.Electrophysiol. 18, 1197-1205 (2007).

66. Lu, Z., et al. Autonomic mechanism for complex fractionated atrial electrograms: evidence by fast fourier transform analysis. J.Cardiovasc. Electrophysiol. 19, 835-842 (2008).

67. Katritsis, D., Giazitzoglou, E., Sougiannis, D., Voridis, E. \& Po, S.S. Complex fractionated atrial electrograms at anatomic sites of ganglionated plexi in atrial fibrillation. Europace. 11, 308-315 (2009).

68. Pokushalov, E., et al. Selective ganglionated plexi ablation for paroxysmal atrial fibrillation. Heart Rhythm 6, 1257-1264 (2009).

69. Nademanee, K., et al. A new approach for catheter ablation of atrial fibrillation: mapping of the electrophysiologic substrate. J Am.Coll.Cardiol. 43, 2044-2053 (2004).

70. Jahangir, A., et al. Long-term progression and outcomes with aging in patients with lone atrial fibrillation: a 30-year follow-up study. Circulation 115, 3050-3056 (2007).

71. Shen, M.J., et al. Patterns of baseline autonomic nerve activity and the development of pacing-induced sustained atrial fibrillation. Heart Rhythm 8, 583-589 (2011).

72. Elvan, A., Huang, X.D., Pressler, M.L. \& Zipes, D.P. Radiofrequency catheter ablation of the atria eliminates pacing-induced sustained atrial fibrillation and reduces connexin 43 in dogs. Circulation 96, 1675-1685 (1997). 
73. Elvan, A., Pride, H.P., Eble, J.N. \& Zipes, D.P. Radiofrequency catheter ablation of the atria reduces inducibility and duration of atrial fibrillation in dogs. Circulation 91, 22352244 (1995).

74. Bauer, A., et al. Effects of circumferential or segmental pulmonary vein ablation for paroxysmal atrial fibrillation on cardiac autonomic function. Heart Rhythm 3, 1428-1435 (2006).

75. Katritsis, D.G., et al. Rapid Pulmonary Vein Isolation Combined with Autonomic Ganglia Modification: A Randomized Study. Heart Rhythm (2010).

76. Ohkubo, K., et al. Combined effect of pulmonary vein isolation and ablation of cardiac autonomic nerves for atrial fibrillation. Int Heart J 49, 661-670 (2008).

77. Bagge, L., et al. Epicardial off-pump pulmonary vein isolation and vagal denervation improve long-term outcome and quality of life in patients with atrial fibrillation. $J$ Thorac Cardiovasc Surg 137, 1265-1271 (2009).

78. Scherlag, B.J., et al. Electrical stimulation to identify neural elements on the heart: their role in atrial fibrillation. J.Interv. Card Electrophysiol. 13 Suppl 1, 37-42 (2005).

79. Scanavacca, M., et al. Selective atrial vagal denervation guided by evoked vagal reflex to treat patients with paroxysmal atrial fibrillation. Circulation 114, 876-885 (2006).

80. Katritsis, D., et al. Anatomic approach for ganglionic plexi ablation in patients with paroxysmal atrial fibrillation. Am J Cardiol 102, 330-334 (2008).

81. Lemery, R., Birnie, D., Tang, A.S., Green, M. \& Gollob, M. Feasibility study of endocardial mapping of ganglionated plexuses during catheter ablation of atrial fibrillation. Heart Rhythm 3, 387-396 (2006).

82. Cummings, J.E., et al. Preservation of the anterior fat pad paradoxically decreases the incidence of postoperative atrial fibrillation in humans. J Am.Coll.Cardiol. 43, 994-1000 (2004). 
83. Hirose, M., Leatmanoratn, Z., Laurita, K.R. \& Carlson, M.D. Partial vagal denervation increases vulnerability to vagally induced atrial fibrillation. J.Cardiovasc.Electrophysiol. 13, 1272-1279 (2002).

84. Kangavari, S., et al. Radiofrequency catheter ablation and nerve growth factor concentration in humans. Heart Rhythm 3, 1150-1155 (2006).

85. okuyama, Y., et al. Sympathetic nerve sprouting induced by radiofrequency catheter ablation in dogs. Heart Rhythm 1, 712-717 (2005).

86. Sakamoto, S., et al. Vagal denervation and reinnervation after ablation of ganglionated plexi. J Thorac Cardiovasc Surg 139, 444-452 (2010).

87. Po, S.S., Nakagawa, H. \& Jackman, W.M. Localization of left atrial ganglionated plexi in patients with atrial fibrillation. J Cardiovasc Electrophysiol 20, 1186-1189 (2009).

88. Oh, S., Choi, E.K. \& Choi, Y.S. Short-term autonomic denervation of the atria using botulinum toxin. Korean Circ J 40, 387-390 (2010).

89. Stavrakis, S., et al. Suppression of atrial fibrillation inducibility by vasostatin-1. Heart Rhythm 8, S265 (2011).

90. Yu, L., et al. Autonomic denervation with magnetic nanoparticles. Circulation 122, 26532659 (2010).

91. Moss, A.J. \& McDonald, J. Unilateral cervicothoracic sympathetic ganglionectomy for the treatment of long QT interval syndrome. N.Engl.J.Med. 285, 903-904 (1971).

92. Schwartz, P.J., et al. Left cardiac sympathetic denervation in the management of highrisk patients affected by the long-QT syndrome. Circulation 109, 1826-1833 (2004).

93. Carpenter, R.J., et al. The acute effects of acupuncture upon autonomic balance in healthy subjects. Am J Chin Med 38, 839-847 (2010). 
94. Abad-Alegria, F., Pomaron, C., Aznar, C., Munoz, C. \& Adelantado, S. Objective assessment of the sympatholytic action of the Nei-Kuan acupoint. Am J Chin Med 29, 201-210 (2001).

95. Huang, S.T., et al. Increase in the vagal modulation by acupuncture at neiguan point in the healthy subjects. Am J Chin Med 33, 157-164 (2005).

96. Li, P., Pitsillides, K.F., Rendig, S.V., Pan, H.L. \& Longhurst, J.C. Reversal of reflexinduced myocardial ischemia by median nerve stimulation: a feline model of electroacupuncture. Circulation 97, 1186-1194 (1998).

97. Nishijo, K., Mori, H., Yosikawa, K. \& Yazawa, K. Decreased heart rate by acupuncture stimulation in humans via facilitation of cardiac vagal activity and suppression of cardiac sympathetic nerve. Neurosci Lett 227, 165-168 (1997).

98. Lomuscio, A., Belletti, S., Battezzati, P.M. \& Lombardi, F. Efficacy of acupuncture in preventing atrial fibrillation recurrences after electrical cardioversion. J Cardiovasc Electrophysiol 22, 241-247 (2011).

99. Chai, C.Y., Huang, T.F. \& Wang, S.C. Mechanisms of cardiac arrhythmias induced by baroceptor reflexes in cats. Am J Physiol 215, 1316-1323 (1968).

100. Zhou, X., Vance, F.L.t., Sims, A.L., Sreenan, C.M. \& Ideker, R.E. Prevention of high incidence of neurally mediated ventricular arrhythmias by afferent nerve stimulation in dogs. Circulation 101, 819-824 (2000).

101. Zhou, X., Wolf, P.D., Smith, W.M., Blanchard, S.M. \& Ideker, R.E. Effects of peroneal nerve stimulation on hypothalamic stimulation-induced ventricular arrhythmias in rabbits. Am J Physiol 267, H2032-2041 (1994).

102. Vanoli, E., et al. Vagal stimulation and prevention of sudden death in conscious dogs with a healed myocardial infarction. Circ.Res. 68, 1471-1481 (1991).

103. Li, S., et al. Low-level vagosympathetic stimulation: a paradox and potential new modality for the treatment of focal atrial fibrillation. Circ Arrhythm Electrophysiol 2, 645651 (2009). 
104. Yu, L., et al. Low-level vagosympathetic nerve stimulation inhibits atrial fibrillation inducibility: direct evidence by neural recordings from intrinsic cardiac ganglia. $J$ Cardiovasc Electrophysiol 22(2010).

105. Sheng, X., et al. Prevention and reversal of atrial fibrillation inducibility and autonomic remodeling by low-level vagosympathetic nerve stimulation. J Am Coll Cardiol 57, 563571 (2011).

106. Shen, M.J., et al. Continuous low-level vagus nerve stimulation reduces stellate ganglion nerve activity and paroxysmal atrial tachyarrhythmias in ambulatory canines. Circulation 123, 2204-2212 (2011).

107. Viskin, S., et al. Circadian variation of symptomatic paroxysmal atrial fibrillation. Data from almost 10000 episodes. European Heart Journal 20, 1429-1434 (1999).

108. Muller, J.E., et al. Circadian variation in the frequency of sudden cardiac death. Circulation 75, 131-138 (1987).

109. Dietrich, S., et al. [A novel transcutaneous vagus nerve stimulation leads to brainstem and cerebral activations measured by functional MRI]. Biomed Tech (Berl) 53, 104-111 (2008).

110. Yu, L., et al. Low level transcutaneous electrical stimulation suprresses atrial fibrillation inducibility. Heart Rhythm 8, S262 (2011).

111. Ardell, J.L. The cardiac neuronal hierarchy and susceptibility to arrhythmias. Heart Rhythm (2010).

112. Ardell, J.L., Cardinal, R., Vermeulen, M. \& Armour, J.A. Dorsal spinal cord stimulation obtunds the capacity of intrathoracic extracardiac neurons to transduce myocardial ischemia. Am J Physiol Regul Integr Comp Physiol 297, R470-477 (2009).

113. Southerland, E.M., et al. Preemptive, but not reactive, spinal cord stimulation mitigates transient ischemia-induced myocardial infarction via cardiac adrenergic neurons. Am J Physiol Heart Circ Physiol 292, H311-317 (2007). 
114. Qin, C., et al. Modulation of neuronal activity in dorsal column nuclei by upper cervical spinal cord stimulation in rats. Neuroscience 164, 770-776 (2009).

115. Wu, M., Linderoth, B. \& Foreman, R.D. Putative mechanisms behind effects of spinal cord stimulation on vascular diseases: a review of experimental studies. Auton Neurosci 138, 9-23 (2008).

116. Olgin, J.E., et al. Effects of thoracic spinal cord stimulation on cardiac autonomic regulation of the sinus and atrioventricular nodes. Journal of Cardiovascular Electrophysiology 13, 475-481 (2002).

117. Lopshire, J.C., et al. Spinal cord stimulation improves ventricular function and reduces ventricular arrhythmias in a canine postinfarction heart failure model. Circulation 120, 286-294 (2009).

118. Lanza, G.A., et al. Spinal cord stimulation for the treatment of refractory angina pectoris: a multicenter randomized single-blind study (the SCS-ITA trial). Pain 152, 45-52 (2011).

119. Andrell, P., et al. Long-term effects of spinal cord stimulation on angina symptoms and quality of life in patients with refractory angina pectoris--results from the European Angina Registry Link Study (EARL). Heart 96, 1132-1136 (2010).

120. Determining the Feasibility of Spinal Cord Neuromodulation for the Treatment of Chronic Heart Failure (DEFEAT-HF), available at http://clinicaltrials.gov/ct2/show/NCT01112579?term=NCT01112579\&rank=1, accessed August 10, 2011. 